\title{
DYNAMICS OF A PLANT-HERBIVORE MODEL WITH A CHEMICALLY-MEDIATED NUMERICAL RESPONSE
}

\author{
LIN WANG, JAMES WATMOUGH, AND FANG YU
}

\begin{abstract}
A system of two ordinary differential equations is proposed to model chemically-mediated interactions between plants and herbivores by incorporating a toxin-modified numerical response. This numerical response accounts for the reduction in the herbivore's growth and reproduction due to chemical defenses from plants. It is shown that the system exhibits very rich dynamics including saddle-node bifurcations, Hopf bifurcations, homoclinic bifurcations and co-dimension 2 bifurcations. Numerical simulations are presented to illustrate the occurrence of multitype bistability, limit cycles, homoclinic orbits and heteroclinic orbits. We also discuss the ecological implications of the resulting dynamics.
\end{abstract}

\section{Introduction}

Ecological systems are fascinating and complex. So are interactions between plants and herbivores. On the one hand, herbivores feed on plants and on the other hand, it is frequently observed that plants can produce chemicals which either are toxic to the herbivores or can inhibit the herbivores' growth and reproduction, preventing the plants from becoming extinct [6]. It has been shown in many works (see, for example, $[1,2,3,4,12,14,17])$ that plant chemical defenses can have a great influence on interactions of plants and herbivores. Hence it is important to take plant chemical defenses into consideration in models of the dynamics of plant-herbivore interactions.

In general, plant chemical defense affects interactions between plants and herbivores from two aspects: (i) directly reducing the amount of plant biomass consumed by herbivores and (ii) inhibiting herbivores' growth and reproduction and increasing their mortality rate [11]. For example, related to the first aspect, tomatoes release jasmonic acid to inhibit the tobacco hornworm's digestion and stop them from getting the needed nitrogen for their own growth [7]. As another example, related to the second aspect, soybeans are resistant to attack by bruchid beetles [13]: bruchid beetle larvae are quickly killed by chemicals released by soybeans shortly after the larvae burrow beneath the seed coat.

When it comes to mathematical modeling, on the first aspect, some recent work has proposed several models with complex toxin-determined functional responses (see $[5,9,10]$ ). Rich dynamics such as oscillations from limit cycles have been observed.

We show that plant chemical defense enriches the dynamics of plant-herbivore interaction via promoting multiple types of saddle-node bifurcations, multiple types of bistability, and homoclinic bifurcations. We first formulate the model and non-dimensionalize it in Section 2. We then show the model is well

Received by the editors 9 October 2020; accepted 28 March 2021; published online 30 March 2021.

2000 Mathematics Subject Classification. Primary 92B05; Secondary 34C23.

Key words and phrases. Plant-Herbivore; Chemical defense; Hopf bifurcation; Homoclinic bifurcation; Heteroclinic orbit.

LW and JW were both supported in part by Natural Science and Engineering Council of Canada (NSERC). 
posed in Section 3. We carry out the detailed mathematical analysis including existence and stability of equilibria in Section 4. Section 5 is devoted to numerically exploring various bifurcations and bistability. A summary and discussion is given in the last section.

\section{Model Formulation}

Let $P(t)$ and $H(t)$ denote the density of plant biomass and the density of herbivore biomass at time $t$, respectively. Assume that the plant grows logistically when its consumer, the herbivore is absent. Plant consumption by the herbivore is modelled by a Holling type II functional response $f$, which takes the form $f(x)=e x /(1+h e x)$, where $e$ represents the rate herbivores encounter plants, and $h$ represents the herbivore's handling time on its food. To account for the plant's chemical defenses, different from [5], we assume that a plant's chemical defense does not directly change the herbivore's consumption behavior, but rather affects the herbivore's growth and reproduction rates. More precisely, we assume that herbivore growth and reproduction rates are functions of the total grazing pressure on the plant, $x=H f(P)$, and that the numerical response, denoted by $g$, has the specific functional form $g(x)=x /\left(1+(x / a)^{2}\right)$. Thus, the numerical response increases with total plant consumption, $x$, when $x$ is low, but decreases if the plant consumption is high. The positive constant $a$ is the scale parameter of the response and is the level of plant consumption for which the numerical response peaks.

Our model is then described by the following coupled differential equations

$$
\begin{aligned}
& \frac{d P(t)}{d t}=R P(t)\left(1-\frac{P(t)}{K}\right)-H(t) f(P(t)), \\
& \frac{d H(t)}{d t}=B g(H(t) f(P(t))-d H(t) .
\end{aligned}
$$

Here $R$ is the intrinsic growth rate and $K$ is the carrying capacity of the plants. The constant $B$ represents the conversion efficiency of consumed plants into new herbivore biomass. The constant $d$ is the death rate for herbivore.

For convenience, we first non-dimensionalize the system by setting

$$
x=\frac{P}{K}, y=\frac{H e}{d}, \hat{t}=t d
$$

and dropping the hats to obtain

$$
\begin{aligned}
& \frac{d x}{d t}=r x(1-x)-\frac{x y}{1+b x}, \\
& \frac{d y}{d t}=-y+\frac{m \frac{x y}{1+b x}}{1+\mu\left(\frac{x y}{1+b x}\right)^{2}},
\end{aligned}
$$

where $r=R / d, b=K h e, m=K B e / d, \mu=K^{2} d^{2} / a^{2}$.

In System (2.2), the key parameter $\mu$ can be interpreted as a measure of the effectiveness of the plant at inhibiting the herbivore's digestion and growth. When $\mu=0$, the model reduces to the RosenzweigMacArthur model [15]. The Rosenzweig-MacArthur model has two boundary equilibria $(0,0)$ and $(1,0)$ and a unique positive equilibrium

$$
\left(u^{*}, v^{*}\right)=\left((m-b)^{-1}, r m\left(1-(m-b)^{-1}\right)\right)
$$

provided $(1+b)<m$. The equilibrium $(0,0)$ is always unstable, the equilibrium $(1,0)$ is stable if $m<1+b$ and is unstable if $m>1+b$. The positive equilibrium can change from being stable to being unstable via a Hopf bifurcation resulting in the appearance of a limit cycle. 


\section{WELL-POSEDNESS OF THE MODEL}

Proposition 3.1. Consider System $(2.2)$ with $(x(0), y(0)) \in \mathbb{R}_{+}^{2}$. There exists a unique solution $(x(t), y(t))$ such that $(x(t), y(t)) \in \mathbb{R}_{+}^{2}$ is bounded.

Proof. The existence and uniqueness of the solution to System (2.2) follows from standard results on ordinary differential equations [18, Theorem 1.1.8]. Note that if $x(0)=0$, then $x(t)=0$ for all $t>0$ and if $y(0)=0$, then $y(t)=0$ for all $t>0$. For any given initial condition $(x(0), y(0)) \in \mathbb{R}_{+}^{2}$, we claim that the unique solution $(x(t), y(t))$ of $(2.2)$ remains non-negative for $t>0$. Suppose this is not true, then there must exist $t_{1}>0$ such that $x(t) \geq 0, y(t) \geq 0$ for $t \in\left[0, t_{1}\right]$ and either $x\left(t_{1}\right)=0$ with $x^{\prime}\left(t_{1}\right)<0$ or $y\left(t_{1}\right)=0$ with $y^{\prime}\left(t_{1}\right)<0$. This is impossible since $\left.\frac{d x}{d t}\right|_{x=0}=\left.\frac{d y}{d t}\right|_{y=0}=0$. This proves that the unique solution $(x(t), y(t)) \in \mathbb{R}_{+}^{2}$ for $t>0$.

Next we show the unique solution is bounded. Consider the equation

$$
X^{\prime}(t)=r X(t)(1-X(t)),
$$

with $X(0)=x(0)$. By a comparison theorem [8, Theorem 1.4.1], we obtain

$$
x(t) \leq X(t)=\frac{1}{1+\left(\frac{1}{x(0)}-1\right) e^{-r t}} \leq M_{1},
$$

where $M_{1}=\max (x(0), 1)$. This shows that $x(t)$ is bounded.

Note that $x(t)$ and $y(t)$ are nonnegative. We then have

$$
\frac{m \frac{x(t) y(t)}{1+b x(t)}}{1+\mu\left(\frac{x(t) y(t)}{1+b x(t)}\right)^{2}} \leq \frac{m}{2 \sqrt{\mu}}=: M_{2} .
$$

Thus

$$
y(t)^{\prime} \leq-y(t)+M_{2}
$$

This implies that

$$
y(t) \leq \max \left(y(0), M_{2}\right)
$$

Thus, $y(t)$ is bounded.

\section{Stability AND BIFURCATIONS}

In this section, we study the dynamics of System (2.2) by considering the stability of the boundary equilibria, $E_{0}=(0,0)$ and $E_{1}=(1,0)$ and the existence, stability and bifurcations of the positive equilibria.

4.1. Stability of boundary equilibria. For the two boundary equilibria, we have the following result on their stability.

Theorem 4.1. For System (2.2), the equilibrium $E_{0}$ is a saddle point with a one dimensional stable manifold and a one dimensional unstable manifold; The herbivore-free equilibrium $E_{1}$ is locally asymptotically stable if

$$
m<1+b
$$

and is a saddle point with a one dimensional stable manifold and a one dimensional unstable manifold if

$$
m>1+b .
$$


Proof. The Jacobian matrix at $E_{0}$ is given by

$$
J_{E_{0}}=\left(\begin{array}{cc}
r & 0 \\
0 & -1
\end{array}\right)
$$

which has two eigenvalues: $\lambda_{1}=r>0$ and $\lambda_{2}=-1<0$. Hence, by [18, Theorem 1.1.3], $E_{0}$ is a saddle point with a one dimensional stable manifold and a one dimensional unstable manifold.

Next we consider the stability of $E_{1}$. The Jacobian matrix at $E_{1}$ is

$$
J_{E_{1}}=\left(\begin{array}{cc}
-r & \frac{-1}{1+b} \\
0 & \frac{m}{1+b}-1
\end{array}\right)
$$

which has two eigenvalues: $\lambda_{1}=-r<0$ and $\lambda_{2}=m /(1+b)-1$. If $m<1+b$ holds, then $\lambda_{2}<0$ and thus $E_{1}$ is locally asymptotically stable, and if $m>1+b$ holds, then $\lambda_{2}>0$. Again, by [18, Theorem 1.1.3], the boundary equilibrium $E_{1}$ is a saddle point with a one dimensional stable manifold and a one dimensional unstable manifold.

Remark 4.2. Using a comparison principle, we can indeed show that $E_{1}$ is globally asymptotically stable if $m<1+b$. The equilibrium $E_{0}$ is unstable and its stable manifold consists of the positive $y$-axis $\{(0, y): y>0\}$. This means that the plant and herbivore can avoid becoming extinct simultaneously unless the initial plant population is zero. The herbivore population goes extinct if the plant is in short supply assuming that the herbivore does not consume other resources.

4.2. Existence of positive equilibria and saddle-node bifurcation. We are more concerned with the coexistence of the plants and the herbivores, which, from mathematics point of view, refers to the existence of positive equilibria for System (2.2).

To explore the existence as well as the stability of positive equilibria, we first establish the following result on the existence of positive equilibria.

Lemma 4.3. System (2.2) admits positive equilibria if and only if $m>1+b$. More specifically, if $m \leq 1+b$, then System (2.2) has no positive equilibria and if $m>1+b$, then System (2.2) has at least one and at most three positive equilibria.

Proof. A positive equilibrium, $\left(x^{*}, y^{*}\right)$, is a positive solution to the following system:

$$
\begin{aligned}
y & =r(1-x)(1+b x), \\
m x & =(1+b x)+\frac{\mu(x y)^{2}}{1+b x} .
\end{aligned}
$$

Substituting (4.3a) into (4.3b) yields

$$
Q(x)=0
$$

with

$$
Q(x)=b x^{5}+(1-2 b) x^{4}+(b-2) x^{3}+x^{2}+\frac{1}{\mu r^{2}}(b-m) x+\frac{1}{\mu r^{2}} .
$$

If $x^{*} \in(0,1)$ is a zero of $Q(x)$, then System $(2.2)$ has an positive equilibrium $\left(x^{*}, y^{*}\right)$ with $y^{*}=$ $r\left(1-x^{*}\right)\left(1+b x^{*}\right)$. It follows from $(4.3 \mathrm{~b})$ that

$$
m x=1+b x+\frac{\mu(x y)^{2}}{1+b x}>1+b x>(1+b) x .
$$

This shows that $m>1+b$ is a necessary condition for System (2.2) to have a positive equilibrium. In fact, it is also a sufficient condition ensuring the existence of at least one positive equilibrium for 


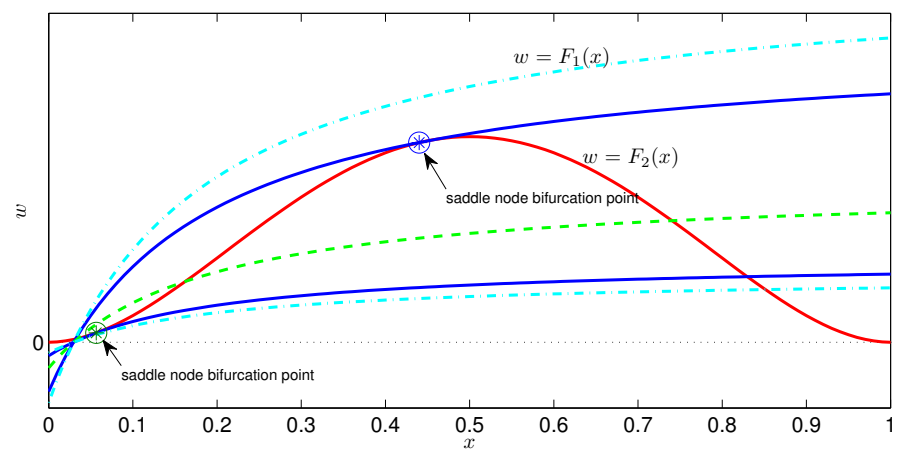

Figure 1. Determination of positive equilibria of System (2.2). Each intersection point of the graphs $w=F_{1}(x)$ and $w=F_{2}(x)$ gives a value of $x^{*}$ for the positive equilibrium $\left(x^{*}, y^{*}\right)$. Here the parameter values are: $r=3.5, b=5.5, m=39.25$. For the five graphs of $w=F_{1}(x)$, from the top to the bottom, the values of $\mu$ are taken as $\mu=4.445,5.445,10.445,19.875,24.875$, respectively.

System (2.2). This follows from the fact that $Q(0)=1 / \mu r^{2}>0$ and $Q(1)=1 /\left(\mu r^{2}\right)(b+1-m)<0$. Moreover, since $b>0$, there exists a sufficiently large $M>1$ such that

$$
Q(-M)<0 \text { and } Q(M)>0 .
$$

This implies that $Q(x)$ always has one negative zero and one positive zero which is larger than 1 . Note that $Q(x)$ can have at most 5 zeros, we can exclude a negative zero and a positive zero which is larger than 1 . This shows that $Q(x)$ can have at least one and at most three zeros on the interval $(0,1)$. That is, System (2.2) has at most three positive equilibria.

Remark 4.4. The herbivore-free equilibrium $E_{1}$ loses the stability at $m=1+b$ and positive equilibria appear when $m>1+b$. This implies that a transcritical bifurcation occurs at $m=1+b$.

Next, we analyze the existence of positive equilibria for System (2.2) graphically. From (4.3), we can derive the following relation:

$$
F_{1}(x):=\frac{1}{\mu r^{2}}\left(\frac{m x}{1+b x}-1\right)=x^{2}(1-x)^{2}=: F_{2}(x) .
$$

An intersection point of the two curves given by $w=F_{1}(x)$ and $w=F_{2}(x)$ for $x \in(0,1)$ determines a positive zero of $Q(x)$ in the interval $(0,1)$. As demonstrated in Figure 1 that $Q(x)=0$ can have either one, or two or three positive roots in $(0,1)$.

Figure 1 implies that System (2.2) may undergo saddle-node bifurcations. Since $\mu$ and $m$ are the parameters associated with herbivore's growth, which may be influenced by plant's chemical defenses, we then focus on how $\mu$ and $m$ affect the dynamics of System (2.2). Suppose all other parameter values $(r$ and $b)$ are fixed, then we can determine a curve in the $\mu-m$ space such that saddle-node bifurcations occur on this curve. We call this curve as a saddle-node bifurcation curve. As it is very difficult to express the $x$ value of the positive equilibrium in terms of the parameters $\mu$ and $m$, we parameterize $\mu$ and $m$ in terms of other parameters and $x$. On the bifurcation curve, we must have $F_{1}(x)=F_{2}(x)$ and 
$F_{1}^{\prime}(x)=F_{2}^{\prime}(x)$, i.e.

$$
\begin{gathered}
\operatorname{chap} \frac{(m-b) x-1}{\mu r^{2}(1+b x)}=x^{2}(1-x)^{2}, \\
\frac{m}{\mu r^{2}(1+b x)^{2}}=2 x(1-x)(1-2 x) .
\end{gathered}
$$

This yields

$$
\begin{aligned}
& \mu=\mu_{s}(x):=\frac{1}{r^{2} x^{2}(1-x) g_{1}(x)}, \\
& m=m_{s}(x):=\frac{2(1-2 x)(1+b x)^{2}}{x g_{1}(x)}
\end{aligned}
$$

for $x \in(0, \bar{x})$, where

$$
g_{1}(x)=-4 b x^{2}+(2 b-3) x+1
$$

and

$$
\bar{x}=\frac{2 b-3+\sqrt{4 b^{2}+4 b+9}}{8 b} \in\left(\frac{1}{3}, \frac{1}{2}\right)
$$

is the unique positive zero of $g_{1}(x)$.

The properties of the two functions $m_{s}(x)$ and $\mu_{s}(x)$ are given in the following lemma.

Lemma 4.5. For $m_{s}(x)$ and $\mu_{s}(x)$ defined in (4.7), we have

$$
\lim _{x \rightarrow 0^{+}} \mu_{s}(x)=\lim _{x \rightarrow \bar{x}^{-}} \mu_{s}(x)=\infty \text { and } \lim _{x \rightarrow 0^{+}} m_{s}(x)=\lim _{x \rightarrow \bar{x}^{-}} m_{s}(x)=\infty .
$$

Moreover, there exists a unique $x_{c} \in(0, \bar{x})$ such that $\mu_{s}^{\prime}\left(x_{c}\right)=m_{s}^{\prime}\left(x_{c}\right)=0, \mu_{s}^{\prime}(x)<0, m_{s}^{\prime}(x)<0$ for $x \in\left(0, x_{c}\right)$ and $\mu_{s}^{\prime}(x)>0, m_{s}^{\prime}(x)>0$ for $x \in\left(x_{c}, \bar{x}\right)$.

Proof. It is straightforward to obtain (4.10), which shows that there exist $\epsilon_{1}>0$ and $\epsilon_{2}>0$ such that both $\mu_{s}(x)$ and $m_{s}(x)$ are decreasing for $x \in\left(0, \epsilon_{1}\right)$ and increasing for $x \in\left(\epsilon_{2}, \bar{x}\right)$. A direct calculation shows that $\operatorname{sgn}\left(\mu_{s}^{\prime}(x)\right)=\operatorname{sgn}\left(m_{s}^{\prime}(x)\right)=\operatorname{sgn}(G(x))$ for $x \in(0, \bar{x})$, where $G(x)$ is given by

$$
G(x):=-10 b x^{3}-(6-12 b) x^{2}+(6-3 b) x-1 .
$$

Therefore, $G(x)<0$ for $x \in\left(0, \epsilon_{1}\right)$ and $G(x)>0$ for $x \in\left(\epsilon_{2}, \bar{x}\right)$. Note that $\lim _{x \rightarrow-\infty} G(x)>0$ and $G(1)=-b-1<0$. This implies that $G(x)$ has one negative zero and two positive zeros located in the intervals $(0, \bar{x})$ and $(\bar{x}, 1)$, respectively. Hence, there exists a unique $x_{c} \in(0, \bar{x})$ such that $\mu_{s}^{\prime}\left(x_{c}\right)=m_{s}^{\prime}\left(x_{c}\right)=G\left(x_{c}\right)=0$ and $\mu_{s}^{\prime}(x)<0, m_{s}^{\prime}(x)<0$ for $x \in\left(0, x_{c}\right)$ and $\mu_{s}^{\prime}(x)>0, m_{s}^{\prime}(x)>0$ for $x \in\left(x_{c}, \bar{x}\right)$.

The above lemma allows us to establish the following result.

Theorem 4.6. Consider System (2.2) with fixed $r$ and $b$. If $m<1+b$, then there are no positive equilibria, while if $m>1+b$, then there exists a saddle-node bifurcation curve and there exists a cusp point at which a codimension-2 bifurcation occurs. Moreover, in the $\mu-m$ space, the locus, $C$, of saddlenode bifurcations has two monotone branches, on which there are exactly two positive equilibria, between which, there are exactly three positive equilibria, and there is a unique positive equilibrium otherwise.

Proof. By Lemma 4.3, we know that there are no positive equilibria if $m<1+b$ and there is at least one positive equilibrium if $m>1+b$. Consider the case with $m>1+b$, it follows from Lemma 4.5 that

$$
C:=\left\{(\mu, m)=\left(\mu_{s}(x), m_{s}(x)\right): x \in(0, \bar{x})\right\}
$$




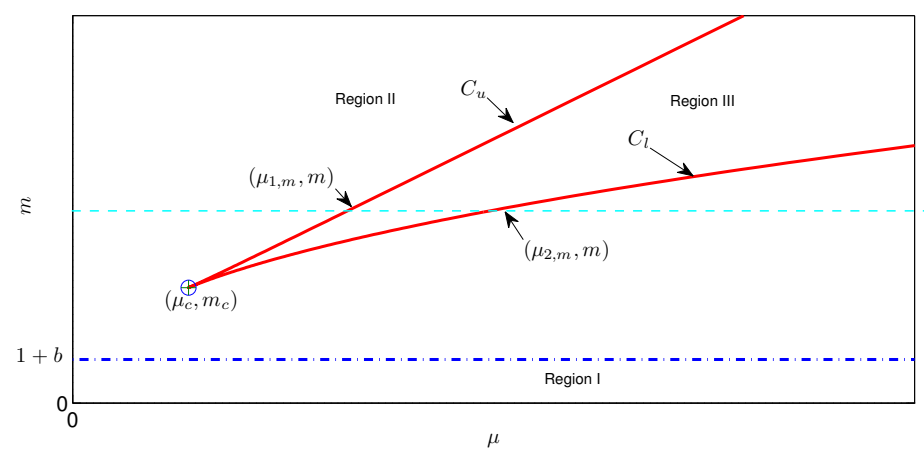

Figure 2. Bifurcation curves of System (2.2) in the $\mu-m$ space. The red curves $C_{u}$ and $C_{l}$ form the saddle-node bifurcation curve; The line given by $m=1+b$ is the transcritical bifurcation curve. Here parameter values used are $r=3.5$ and $b=3.5$.

defines a curve in the $\mu-m$ space. Define two branches $C_{u}$ and $C_{l}$ as

$$
C_{u}=\left\{(\mu, m)=\left(\mu_{s}(x), m_{s}(x)\right): x \in\left(x_{c}, \bar{x}\right)\right\}
$$

and

$$
C_{l}=\left\{(\mu, m)=\left(\mu_{s}(x), m_{s}(x)\right): x \in\left(0, x_{c}\right)\right\}
$$

then

$$
C=C_{u} \cup C_{l} \cup\{\mathbf{C P}\}
$$

with

$$
\mathbf{C P}=\left(\mu_{c}, m_{c}\right)=\left(\mu_{s}\left(x_{c}\right), m_{s}\left(x_{c}\right)\right),
$$

where $x_{c}$ is given in Lemma 4.5. Lemma 4.5 indicates that the two branches are monotone in the sense that $\mu_{s}(x)$ and $m_{s}(x)$ are monotone in $x$ for $(\mu, m)$ on $C_{u}$ and $C_{l}$,respectively. If $(\mu, m) \in C$, then the graphs of $F_{1}(x)$ and $F_{2}(x)$ intersect tangentially at some point, say $\left(x^{*}, F_{1}\left(x^{*}\right)\right)$, which determines an additional positive equilibrium $\left(x^{*}, y^{*}\right)$ for System (2.2). That is, there are exactly two positive equilibria if $(\mu, m)$ is located on $C$. If $(\mu, m)$ is located between $C_{u}$ and $C_{l}$ (see Region III in Figure 2), then the curve given by $w=F_{1}(x)$ (locally) lies below the above mentioned point $\left(x^{*}, F_{1}\left(x^{*}\right)\right)$ and hence there are two intersection points for the graphs of $F_{1}(x)$ and $F_{2}(x)$ resulting in two additional positive equilibria of System (2.2). That is, there are exactly three positive equilibria when $(\mu, m)$ is in Region III. If $(\mu, m)$ is located on the other side of the curve $C$, then the curve given by $w=F_{1}(x)$ (locally) lies above the point $\left(x^{*}, F_{1}\left(x^{*}\right)\right)$ and hence there is no intersection point for the graphs of $F_{1}(x)$ and $F_{2}(x)$. Consequently, System (2.2) does not admit any positive equilibria near $\left(x^{*}, y^{*}\right)$ and there is exactly one positive equilibrium. This shows that there exists a saddle-node bifurcation curve which consists of $C_{u}$ and $C_{l}$. Note that the two monotone branches $C_{u}$ and $C_{l}$ meet tangentially at $\mathbf{C P}$ with $\mu_{s}^{\prime}\left(x_{c}\right)=m_{s}^{\prime}\left(x_{c}\right)=0$. This indicates that $\mathbf{C P}$ is a cusp point, at which a codimension-2 bifurcation occurs [16].

4.3. Stability of positive equilibria. Using (4.3), we can express the entries of the Jacobian matrix $J$ at a positive equilibrium $(x, y)$ in terms of $x$ as

$$
J=\left[\begin{array}{ll}
a_{11} & a_{12} \\
a_{21} & a_{22}
\end{array}\right]
$$


with

$$
\begin{aligned}
& a_{11}=r-2 r x-\frac{r(1-x)}{1+b x}, \quad a_{12}=-\frac{x}{1+b x}, \\
& a_{21}=\frac{r(1-x)(2+(2 b-m) x)}{m x^{2}} \text {, and } a_{22}=-2\left(1-\frac{1+b x}{m x}\right) .
\end{aligned}
$$

The corresponding characteristic equation is then given by

$$
\lambda^{2}-T(x) \lambda+D(x)=0,
$$

with

$$
T(x)=a_{11}+a_{22}=-2+r-2 r x+\frac{2(1+b x)}{m x}-\frac{r(1-x)}{1+b x}
$$

and

$$
D(x)=a_{11} a_{22}-a_{12} a_{21}=\frac{r\left(2(1-2 x)(1+b x)^{2}-m x g_{1}(x)\right)}{m x(1+b x)},
$$

where $g_{1}(x)$ is defined in (4.8).

Lemma 4.7. Let $(x, y)$ be a positive equilibrium of System (2.2). If $b \leq 1$ or $b>1$ and $m \leq$ $b(b+1) /(b-1)$, then $T(x)<0$; If $b>1$ and $m>b(b+1) /(b-1)$, then there exists an $x_{H} \in$ $\left((m-b)^{-1},(b-1) /(2 b)\right)$ such that $T\left(x_{H}\right)=0$, and $T(x)<0$ for $x \in\left(x_{H}, 1\right)$.

Proof. Since $(x, y)$ is a positive equilibrium of System $(2.2)$, then $m>1+b$ and $x \in\left((m-b)^{-1}, 1\right)$. Note that $T(x)$ can be rearranged as

$$
T(x)=r \frac{x(b-1-2 b x)}{1+b x}-2 \frac{(m-b) x-1}{m x} .
$$

Note that $((m-b) x-1) /(m x)>0$. Thus, if $b \leq 1$ or $b>1$ and $m \leq b(b+1) /(b-1)$, then $b-1-2 b x<$ 0 and hence $T(x)<0$. Assume $b>1$ and $m>b(b+1) /(b-1)$. Note that $T\left((m-b)^{-1}\right)>0$ and $T(x)<0$ for $x \geq(b-1) /(2 b)$. Then there must exit an $x_{H} \in\left((m-b)^{-1},(b-1) /(2 b)\right)$ such that $T\left(x_{H}\right)=0$, and $T(x)<0$ for $x \in\left(x_{H}, 1\right)$.

Lemma 4.8. For fixed $r$ and $b$, at any positive equilibrium $(x, y)$, we have the following conclusions.

(i) if $1+b<m<m_{c}$, then $D(x)>0$ for any $\mu \geq 0$;

(ii) if $m \geq m_{c}$, then for each $m$, as $\mu$ changes, there are two saddle-node bifurcation values $x_{2, m}^{*}<x_{1, m}^{*}$ with $D\left(x_{i, m}^{*}\right)=0(i=1,2), D(x)<0$ for $x \in\left(x_{2, m}^{*}, x_{1, m}^{*}\right)$ and $D(x)>0$ otherwise.

Proof. If $x \in(0, \bar{x})$, then $g_{1}(x)>0$ and we can rewrite $D(x)$ as

$$
D(x)=\frac{r g_{1}(x)\left(m_{s}(x)-m\right)}{m(1+b x)},
$$

where $m_{s}(x)$ is defined in (4.7b). If $1+b<m<m_{c}$, then $m_{s}(x)>m$ for $x \in(0, \bar{x})$. Thus $D(x)>0$ for $x \in(0, \bar{x})$. Using (4.3), we can also write $D(x)$ as

$$
D(x)=\frac{r}{m x} D_{1}(x),
$$

where

$$
D_{1}(x)=1-x-\mu r^{2} x^{2}(1-x)^{2} g_{1}(x) .
$$

Note that $g_{1}(x) \leq 0$ for $x \in(\bar{x}, 1)$, we see that $D_{1}(x)>0$ for $x \in[\bar{x}, 1)$. This proves case (i).

In case (ii), if $m>m_{c}$, then for each $m$, according to Theorem 4.6, there exist two saddle-node bifurcation values $x_{2, m}^{*}<x_{1, m}^{*}$ such that $\left(\mu_{1, m}, m\right) \in C_{u}$ and $\left(\mu_{2, m}, m\right) \in C_{l}$, where $\mu_{1, m}=\mu_{s}\left(x_{1, m}^{*}\right)$, 
$\mu_{2, m}=\mu_{s}\left(x_{2, m}^{*}\right)$ and $m=m_{s}\left(x_{i, m}\right)^{*}$ for $i=1,2$. Thus $D\left(x_{1, m}^{*}\right)=D\left(x_{2, m}^{*}\right)=0$. On the other hand, we can express $D(x)$ as

$$
D(x)=\frac{r g_{2}(x)}{m x(1+b x)},
$$

where $g_{2}(x)=\left(4 b m-2 b^{2}\right) x^{3}+\left(2 b^{2}-4 b-(2 b-3) m\right) x^{2}+(4 b-2-m) x+2$. Note that $4 b m-2 b^{2}>0$, $g_{2}(0)=2>0$, then the cubic function $g_{2}(x)$ must have a negative zero and two positive zeros $x_{1, m}^{*}$ and $x_{2, m}^{*}$. By the property of cubic functions, we know that $D(x)<0$ for $x \in\left(x_{2, m}^{*}, x_{1, m}^{*}\right)$ and $D(x)>0$ for $x \in\left(0, x_{2, m}^{*}\right) \cup\left(x_{1, m}^{*}, 1\right)$.

Similarly, we can prove the following lemma.

Lemma 4.9. For fixed $r$ and $b$, at any positive equilibrium $(x, y)$, we have

(iii) if $\mu \leq \mu_{c}$ and $m>1+b$, then $D(x)>0$;

(iv) if $\mu>\mu_{c}$ and $m>1+b$, then for each $\mu$, as $m$ changes, there are two saddle-node bifurcation values $x_{i, \mu}^{*}, i=1,2$ at which $D(x)=0, D(x)<0$ when $x$ is between these two values, and $D(x)>0$ otherwise.

Our main result on stability of positive equilibria is presented below.

Theorem 4.10. Consider System (2.2). If System (2.2) has a unique positive equilibrium $\left(x^{*}, y^{*}\right)$, then it is stable provided $T\left(x^{*}\right)<0$; If System (2.2) has three positive equilibria $\left(x_{i}^{*}, y_{i}^{*}\right)$ with $x_{1}^{*}<x_{2}^{*}<x_{3}^{*}$, then the middle equilibrium $\left(x_{2}^{*}, y_{2}^{*}\right)$ is a saddle point, the other two equilibria can be stable or unstable nodes. In both cases, if at a positive equilibrium $\left(x_{H}, y_{H}\right)$ such that $T\left(x_{H}\right)=0$ and $D\left(x_{H}\right)>0$, then a Hopf bifurcation occurs at this positive equilibrium.

Proof. The proof follows directly from Lemmas 4.7-4.9.

\section{Rich DYNAMICS: NUMERICAL EXPLORATION}

For each positive equilibrium $\left(x^{*}, y^{*}\right)$, since we cannot explicitly express $x^{*}$ in terms of system parameters, we are not able to determine the sign of $T\left(x^{*}\right)$. In this section, we present some numerical simulations to explore possible dynamics that may emerge in System (2.2).

We take $\mu$ as the bifurcation parameter. According to Theorem 4.6, for each $m>m_{c}$, there are two saddle-node bifurcation values, $\mu_{1, m}<\mu_{2, m}$, which give two saddle-node bifurcation points $S N_{1}=$ $\left(x_{1, m}^{*}, y_{1, m}^{*}\right)$ and $S N_{2}=\left(x_{2, m}^{*}, y_{2, m}^{*}\right)$ satisfying $x_{1, m}^{*}>x_{2, m}^{*}$ (See Theorem 4.6 (ii)). These two saddlenode bifurcation points divide the set of equilibria into three subsets: $E_{U}=\left\{\left(x^{*}, y^{*}\right): x^{*}>x_{1, m}^{*}\right\}$, $E_{M}=\left\{\left(x^{*}, y^{*}\right): x^{*} \in\left(x_{2, m}^{*}, x_{1, m}^{*}\right)\right\}$, and $E_{L}=\left\{\left(x^{*}, y^{*}\right): x^{*}<x_{2, m}^{*}\right\}$. Moreover, by Theorem 4.10, every positive equilibrium $\left(x^{*}, y^{*}\right) \in E_{M}$ is a saddle point. Since System (2.2) admits three positive equilibria when $\mu \in\left(\mu_{1, m}, \mu_{2, m}\right)$, we see that a pair of saddle-node bifurcations occurs at $S N_{1}$ and $S N_{2}$. Depending on the stability of the positive equilibria, we may have the following possible scenarios.

Scenario I. This corresponds to the case where $\left(x^{*}, y^{*}\right)$ is locally asymptotically stable for $\left(x^{*}, y^{*}\right) \in$ $E_{U} \cup E_{L}$. That is, a stable node and a saddle point bifurcate from both saddle-node points $S N_{1}$ and $\mathrm{SN}_{2}$. Such a bifurcation diagram is shown in Figure 3, with a corresponding phase portrait given in Figure 4. In this case, System (2.2) exhibits Type-I bistability: a stable positive equilibrium coexists with another stable positive equilibrium (see Figure 4).

Scenario II. In this case, there exists a Hopf bifurcation point $H_{P}=\left(x_{H}, y_{H}\right) \in E_{L}$ and $\left(x^{*}, y^{*}\right)$ is locally asymptotically stable for $\left(x^{*}, y^{*}\right) \in E_{U} \cup E_{L S}$ and $\left(x^{*}, y^{*}\right)$ is unstable for $\left(x^{*}, y^{*}\right) \in E_{L U}$. Here 


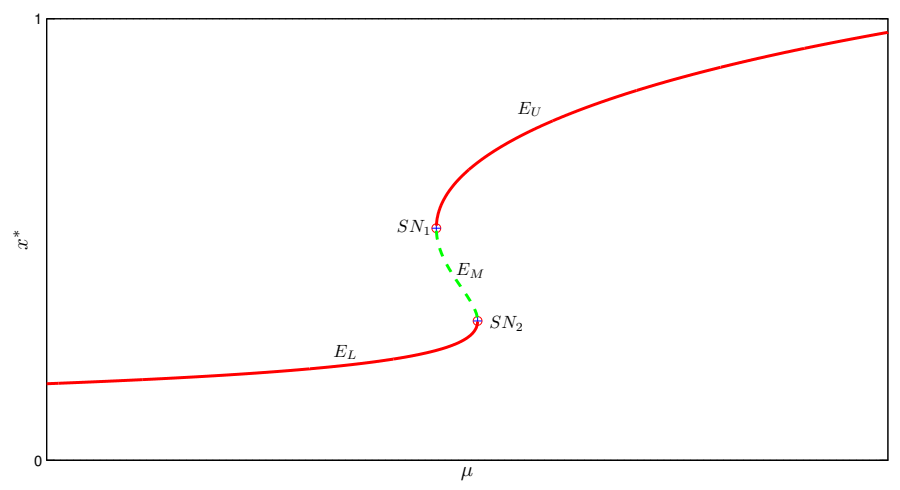

FiguRE 3. Bifurcation diagram associated with Scenario I. Here parameter values used are $r=3.5, b=0.5$ and $m=15$.

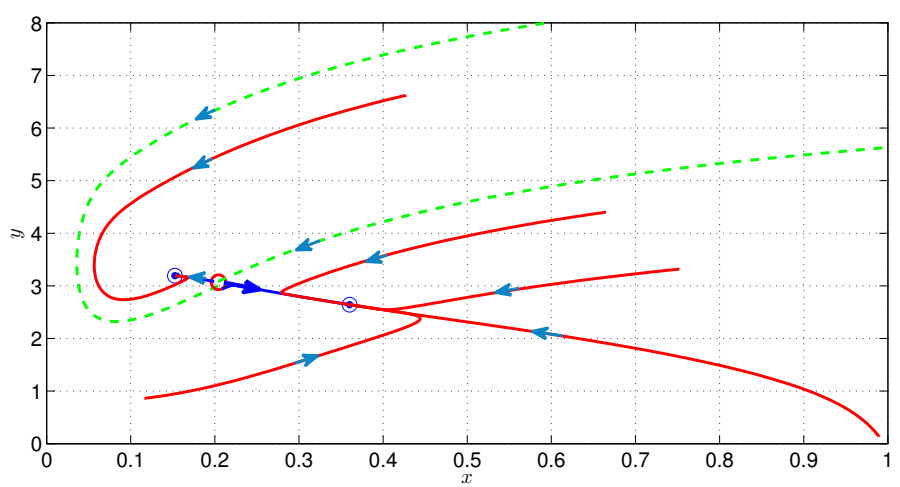

Figure 4. A phase portrait of System (2.2) illustrating a Type-I bistability (Two stable nodes coexist). Here parameter values used are $r=3.5, b=1.5, m=20$ and $\mu=5.5$. The two stable equilibria are separated by stable manifolds of the saddle point (dashed curves).

$E_{L S}:=\left\{\left(x^{*}, y^{*}\right): x^{*} \in\left(x_{H}, x_{2, m}^{*}\right)\right\}$ and $E_{L U}:=\left\{\left(x^{*}, y^{*}\right): x^{*}<x_{H}\right\}$. Note that a stable node and a saddle point bifurcate from both the saddle-node points $S N_{1}$ and $S N_{2}$, but as $\mu$ decreases, the positive equilibrium on the node branch of $S N_{2}$ becomes unstable due to the occurrence of Hopf bifurcation. See Figure 5 for a typical bifurcation diagram. Consequently, besides Type-I bistability, Type-II bistability emerges: a stable positive equilibrium coexists with a stable limit cycle (See Figure 6).

Scenario III. In this case, the positive equilibrium $\left(x^{*}, y^{*}\right)$ is locally asymptotically stable for $\left(x^{*}, y^{*}\right) \in E_{U}$ and $\left(x^{*}, y^{*}\right)$ is unstable for $\left(x^{*}, y^{*}\right) \in E_{L}$. A stable node and a saddle-point bifurcate from the saddle-node bifurcation point $S N_{1}$, while an unstable node and a saddle-point bifurcate from the saddle-node bifurcation point $S N_{2}$. A bifurcation diagram is presented in Figure 7 . In the case that the system admits three positive equilibria, $E_{1} \in E_{L}, E_{2} \in E_{M}$ and $E_{3} \in E_{U}$, multiple heteroclinic orbits exist: one from $(1,0)$ to $E_{3}$, one from $E_{1}$ to $E_{3}$ and two from $E_{2}$ to $E_{3}$. A phase portrait is shown in Figure 8. 


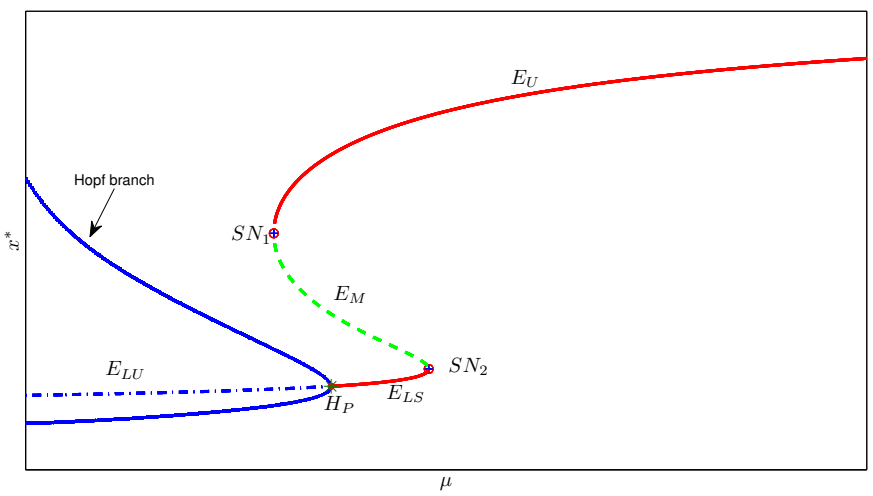

FIGURE 5. Bifurcation diagram associated with Scenario II. Here parameter values used are $r=3.5, b=3.5$ and $m=20$.

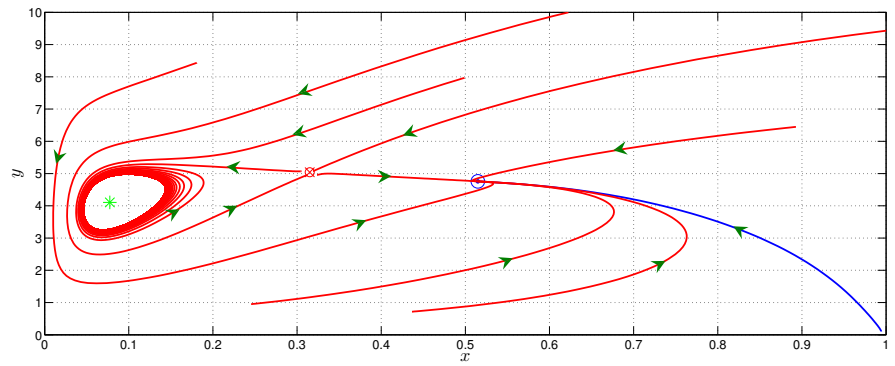

Figure 6. A phase portrait of System (2.2) exhibiting a Type-II bistability (A stable node and a stable limit cycle coexist). Here parameter values used are $r=3.5, b=3.5$, $m=20$ and $\mu=3.5$.

Scenario IV. In this case there exists a Hopf bifurcation point $\left(x_{H}, y_{H}\right) \in E_{U}$ and $\left(x^{*}, y^{*}\right)$ is locally asymptotically stable for $\left(x^{*}, y^{*}\right) \in E_{U S}$ and $\left(x^{*}, y^{*}\right)$ is unstable for $\left(x^{*}, y^{*}\right) \in E_{L} \cup E_{U U}$, where $E_{U S}:=\left\{\left(x^{*}, y^{*}\right): x^{*}>x_{H}\right\}$ and $E_{U U}:=\left\{\left(x^{*}, y^{*}\right): x^{*} \in\left(x_{1, m}^{*}, x_{H}\right)\right\}$. As shown in Figure 9, locally, an unstable node and a saddle-point bifurcate from both saddle-node points $S N_{1}$ and $S N_{2}$.

Homoclinic bifurcation and type-III bistability. In Scenario II, there exists a Hopf bifurcation point $H_{P}$ on $E_{L}$ corresponding a critical value $\mu_{H} \in\left(\mu_{1, m}, \mu_{2, m}\right)$. This results in a stable limit cycle when $\mu$ is smaller than but nearby $\mu_{H}$. Depending on the values of the system parameters, the continuation of the limit cycle induced from Hopf bifurcation will be terminated via a homoclinic bifurcation, where the limit cycle branch touches a saddle point on $E_{M}$. On the other hand, when $\mu<\mu_{1, m}$, System (2.2) has only one unstable positive equilibrium $\left(x^{*}, y^{*}\right) \in E_{L U}$ and both boundary equilibria are unstable. Since it is a two dimensional system, and the solutions are nonnegative and bounded, there must exist at least one limit cycle around the positive equilibrium $\left(x^{*}, y^{*}\right)$. As $\mu$ increases, it is possible for the limit cycle branch to touch the saddle point resulting in a homoclinic 


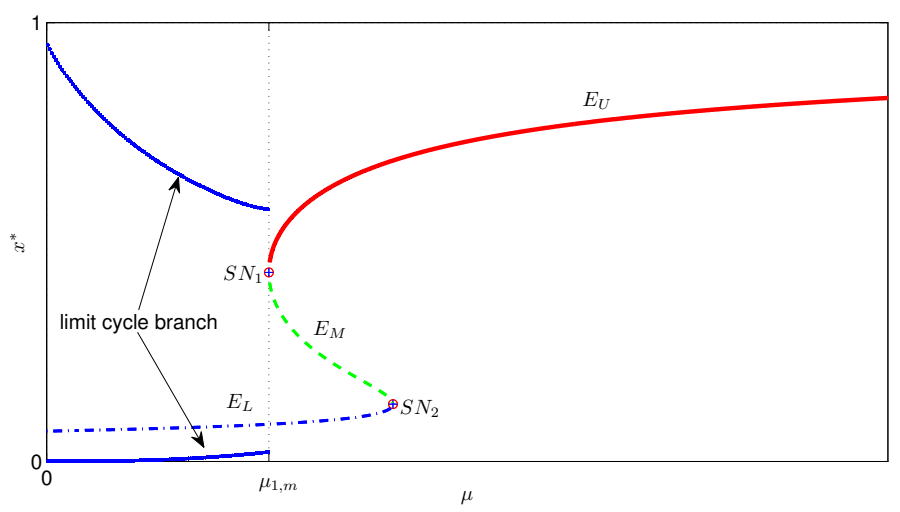

FiguRE 7. Bifurcation diagram associated with Scenario III. Here parameter values used are $r=3.5, b=5.5$ and $m=20$.

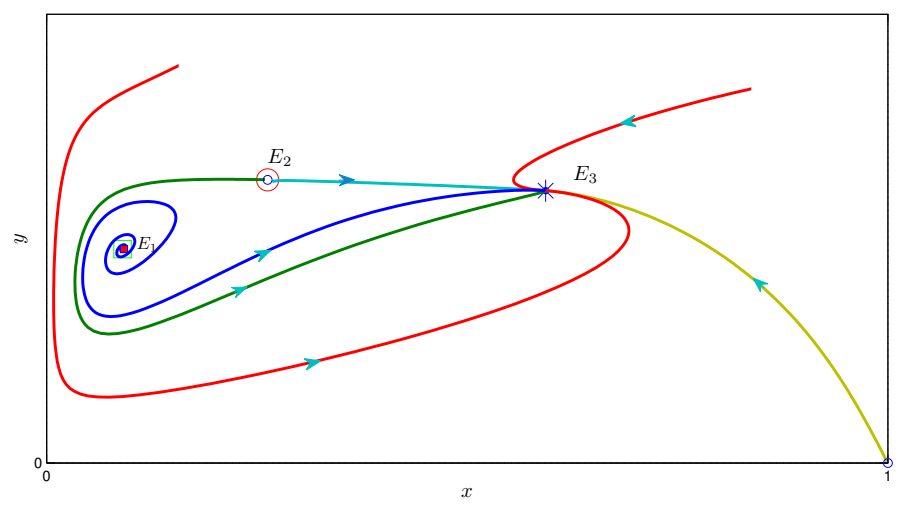

Figure 8. A phase portrait of System (2.2) exhibiting the existence of multiple heteroclinic orbits. Here parameter values used are $r=3.5, b=5.5, m=20$ and $\mu=2.5$.

bifurcation. This type of dynamics induces the type-III bistability: a stable positive equilibrium coexists with a half-stable homoclinic orbit.

We take the parameter values as $r=3.5, b=5.5$ and $m=39.25$. As shown in Figure 10, there exists a critical value $\mu_{H} \approx 16.52$ at which a Hopf bifurcation occurs resulting a stable limit cycle. The continuation of the stable limit cycle terminates as $\mu$ is decreased to $\mu_{2}^{\text {hom }} \approx 11.07$ at which a homoclinic bifurcation occurs resulting a homoclinic orbit. Moreover, there exists another critical value $\mu_{1}^{\text {hom }} \approx 7.02$ at which a homoclinic bifurcation occurs resulting another homoclinic orbit. A representative phase portrait is depicted in Figure 11.

Codimension-2 bifurcations Regarding both $\mu$ and $m$ as bifurcation parameters, codimension-2 bifurcations including Bogdanov-Takens bifurcation and cusp point are also possible in our model. To verify this, we use the Matcont package to plot the bifurcation curves in the $\mu-m$ plane to get Figure 12 . 


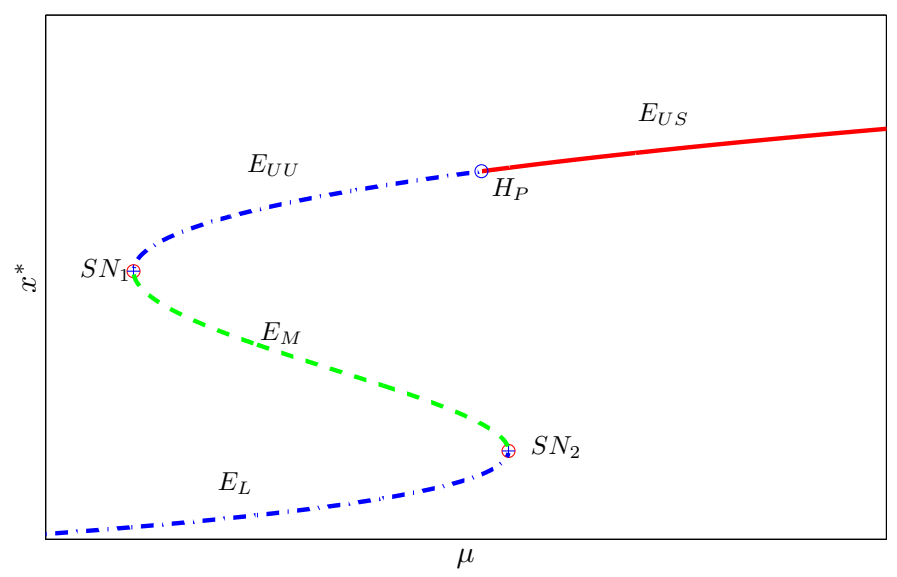

Figure 9. Bifurcation diagram associated with Scenario IV. A subcritical Hopf bifurcation occurs at $H_{P}$. Here parameter values used are: $r=20, b=5.8$ and $m=13.5$.

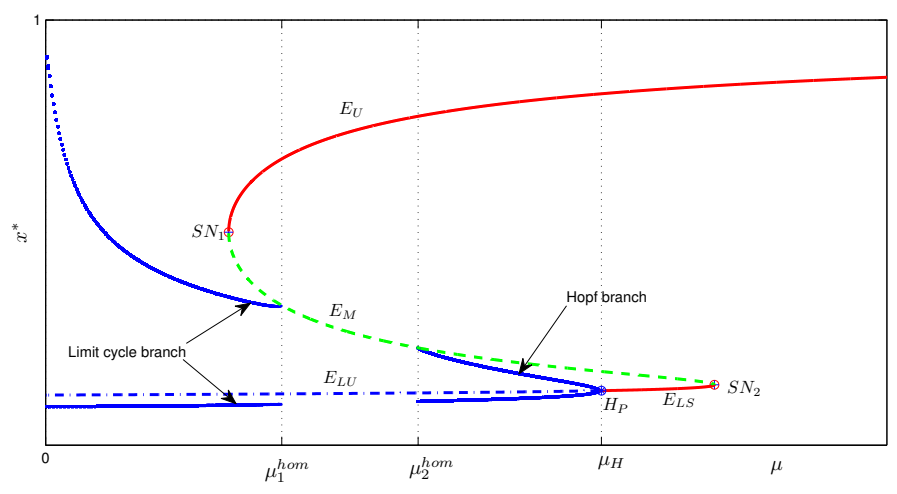

Figure 10. Bifurcation diagram of System (2.2) with a Hopf bifurcation and two homoclinic bifurcations.

As shown in Figure 12, between the two saddle-node bifurcation curves, there is a Hopf bifurcation curve. In addition, there are a cusp point $(0.90,13.11)$ and two Bogdanov-Takens points $(1.05,13.80)$ and $(7.09,26.29)$ on both the Hopf bifurcation curve and the saddle-node bifurcation curves.

\section{Summary AND Discussion}

In this paper we have proposed a new plant-herbivore model that includes a toxin-determined numerical response to account for the effect of plant's chemical defenses. We have shown that the toxindetermined numerical response can induce very rich dynamics including saddle-node bifurcations, Hopf bifurcation, homoclinic and Bogdanov-Takens bifurcation. This results in three types of bistability: Type-I bistability (one stable equilibrium coexists with another stable equilibrium); Type-II bistability (one stable equilibrium coexists with one stable limit cycle) and Type-III bistability (one stable equilibrium coexists with one half stable homoclinic orbit). 


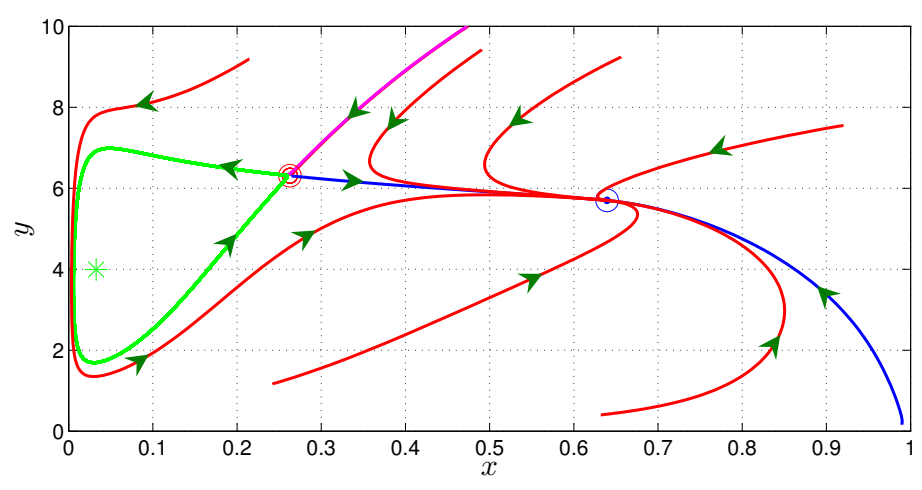

Figure 11. Phase portrait of System (2.2) exhibiting a Type-III bistability. Here parameter values used are $r=3.5, b=5.5, m=39.25$ and $\mu=7.02$.

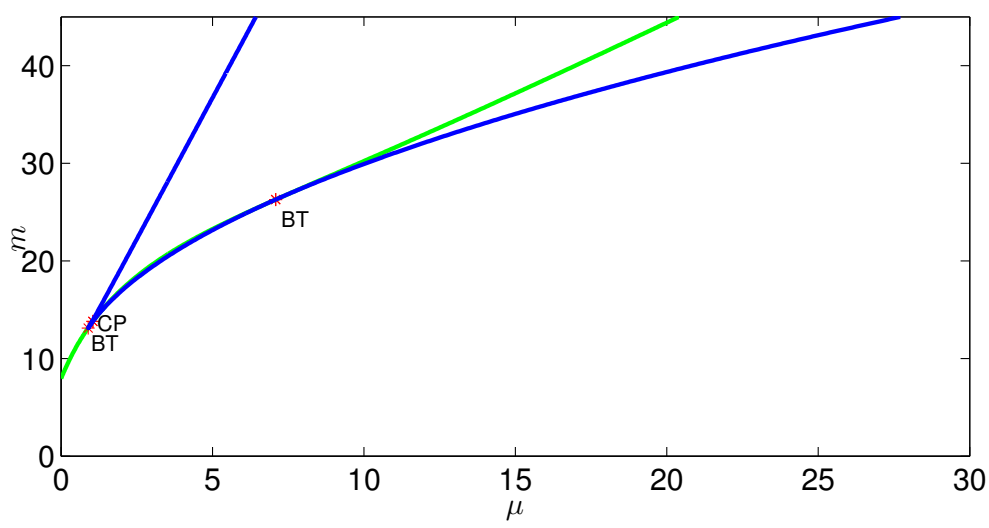

FiguRE 12. Saddle-node bifurcation curve and Hopf bifurcation curve in System (2.2): CP-cusp point; BT-Bogdanov-Takens point. Here parameter values used are: $r=3.5$, $b=5.5$.

The model's key parameter $\mu$ measures the effectiveness of the plant's inhibiting effect on the herbivore's growth and reproduction. In principle, $\mu$ increases the plant's ability to maintain a relatively high population density preventing it from extinction. Depending on other model parameters, various dynamic behaviours are possible. For instance, Figure 10 represents a bifurcation diagram for certain combination of parameters. When there is no chemical defense, that is, $\mu=0$, the system has a unique stable limit cycle induced by the Holling type II functional response. Both the plant population and the herbivore population oscillate about the unique positive equilibrium. If $\mu>0$, then there are 6 intervals in which dynamics are different: Case (i), $\mu \in\left(0, \mu_{1, m}\right)$. There is a unique positive equilibrium, which is unstable. The solutions tend to a stable limit cycle. As $\mu$ increases, the amplitude of the limit cycle decays. Case (ii), $\mu \in\left(\mu_{1, m}, \mu_{1}^{\text {hom }}\right)$. There are three positive equilibria. Among them, one is a stable node, one is a saddle point, and the other is an unstable node. The solutions with initial conditions different from these equilibria approach either the stable node on $E_{U}$ or a stable limit cycle enclosing the unstable node on $E_{L U}$. Case (iii), $\mu \in\left(\mu_{1}^{h o m}, \mu_{2}^{h o m}\right)$. There are three positive equilibria and the solutions approach the stable node on $E_{U}$. In addition, there are two heteroclinic orbits from the saddle 
point to the stable node and one heteroclinic orbit from the unstable node to the stable node. Case (iv), $\mu \in\left(\mu_{2}^{\text {hom }}, \mu_{H}\right)$. As in case (ii), the solutions approach either the stable node on $E_{U}$ or a stable limit cycle enclosing the unstable node on $E_{L U}$. Case (v), $\mu \in\left(\mu_{H}, \mu_{2, m}\right)$. The solutions approach either the stable node on $E_{U}$ or the stable node on $E_{L S}$. Case (vi), $\mu>\mu_{2, m}$. There is a unique positive equilibrium to which the solutions approach. Biologically, in case (i), the plant population undergoes periodic fluctuations with decaying amplitudes. In cases (ii) and (iv), the outcome is initial condition dependent: depending on the initial population, the plant population either maintains at a constant level or fluctuates about a lower level. In cases (iii) and (vi), the plant population stabilizes at a constant level, which is higher than the case with $\mu=0$. But case (iii) has a threshold (the stable manifold of the saddle point) for perturbations from equilibrium which lead to catastrophic plant-populations declines. In case (v), depending on the initial population, the plant population maintains at either a high level (corresponding to the equilibrium on the upper branch) or a low level (corresponding to the equilibrium on the lower branch).

Bistability exhibited in our model can be used to design suitable control strategies for resource management purpose. For instance, in the case of Type-II bistability, a stable positive equilibrium on the upper equilibrium branch coexists with a stable limit cycle that oscillates about an unstable equilibrium located on the lower equilibrium branch. If the plant population is in the basin of attraction of the limit cycle, and it is required to increase the plant population and avoid sustained oscillations, then a strategy that can shift the solution to the basin of the attraction of the stable equilibrium should be sufficient.

\section{ACKNowledgements}

The authors are very grateful to the handling editor and the reviewers' comments and suggestions.

\section{REFERENCES}

[1] J.P Bryant, F S.Chapin III, D.R Klein, Carbon/nutrient balance of boreal plants in relation to vertebrate herbivory, Oikos 40(1983), 357-368.

[2] J.P Bryant, P.B Reichardt, TP Clausen, Chemically mediated interactions between woody plants and browsing mammals, J. Range Manag. 45(1992), 18-24.

[3] J.P Bryant, R.K. Swihart, P.B. Reichardt, L. Newton, Biogeography of woody plant chemical defense against snowshoe hare browsing: comparison of Alaska and eastern North America, Oikos 70(1994), 385-395.

[4] P.D Coley, J.P Bryant, F S. Chapin III, Resource availability and plant antiherbivore defense, Science(Washington) 230(1985), 895-899.

[5] Z. Feng, R. Liu, D. DeAngelis, Plant-herbivore interactions mediated by plant toxicity, Theor. Pop. Biol. 73(2008), 449-459.

[6] L.R. Fox, Defense and dynamics in plant-herbivore systems, Amer. Zool. 21(1981), 853-864.

[7] A.L. Iverson, L.R. Iverson and S. Eshita, The effects of surface-applied jasmonic and salicylic acids on caterpillar growth and damage to tomato plants, OHIO J. Sci. 101(2001), 90-94.

[8] V. Lakshmikantham and S. Leela, Differential and Integral Inequalities, Academic Press, 1969.

[9] Y. Li, Z. Feng, R. Swihart, J. Bryant, N. Huntly: Modeling the impact of plant toxicity on plant-herbivore dynamics, J. Dynam. Differential Equations 18(2006), 1021-1042.

[10] R. Liu, Z. Feng, H. Zhu, D.L. DeAngelis: Bifurcation analysis of a plant-herbivore model with toxin-determined functional response, J. Differential Equations 245(2008), 442-467.

[11] W.W. Murdoch, Community structure, population control, and competition-A critique, Am. Nat. 100(1966), 219-226.

[12] R.T. Palo, C.T. Robbins, Plant defenses against mammalian herbivory, CRC Press, New York, 1991.

[13] R.E. Ricklefs, The Economy of Nature, Freeman and Company, New York, 2010.

[14] G.A. Rosenthal, M.R. Berenbaum, Herbivores: Their interactions with secondary plant metabolites: Ecological and Evolutionary Processes, Elsevier, 1992. 
[15] M. Rosenzweig and R. MacArthur, Graphical representation and stability condition of predator-prey interaction, Am. Nat. 97(1963), 209-223.

[16] S.H. Strogatz, Nonlinear Dynamics and Chaos: with Applications to Physics, Biology, Chemistry and Engineering, Perseus Books Group, New York, 2001.

[17] J.J. Villalba, F.D. Provenza, J.P Bryant, Consequences of the interaction between nutrients and plant secondary metabolites on herbivore selectivity: benefits or detriments for plants?, Oikos 97(2002), 282-292.

[18] S. Wiggins, Introduction to Applied Nonlinear Dynamical Systems and Chaos, Springer, 1990.

Corresponding author. Department of Mathematics and Statistics, University of New Brunswick, FredERICTON, NB, E3B 5A3, CANADA

E-mail address: 1wang2@unb.ca

Department of Mathematics and Statistics, University of New Brunswick, Fredericton, NB, E3B 5A3, CANADA

E-mail address: watmough@unb.ca

Department of Mathematics and Statistics, University of New Brunswick, Fredericton, NB, E3B 5A3, CANADA

E-mail address: yufang77@gmail.com 